\title{
Linear envelope model for multicharge state linac
}

\author{
Z. $\mathrm{He}^{*}$ \\ Facility for Rare Isotope Beams, Michigan State University, East Lansing, Michigan 48824, USA \\ and Tsinghua University, Haidian District, Beijing 100084, China \\ Y. Zhang, J. Wei, and Z. Liu \\ Facility for Rare Isotope Beams, Michigan State University, East Lansing, Michigan 48824, USA \\ R. M. Talman \\ Cornell University, Ithaca, New York 14850, USA \\ (Received 11 October 2013; published 7 March 2014)
}

\begin{abstract}
The traditional linear envelope model is widely used in linac design and on-line tuning. However, for the simultaneous acceleration of multicharge states, the associated transfer matrix acts differently on each species with different charge to mass ratios and the traditional linear envelope model cannot be utilized. A direct way to handle multicharge state acceleration is by using multiparticle tracking, which can be high in model detail, but is typically lacking in computational efficiency to the extent where it is not suitable for on-line beam tuning of a linac. In this paper, a new approach of adapting a conventional linear envelope model to multicharge state acceleration is developed. The lattice of the proposed Facility for Rare Isotope Beams (FRIB) is used to test this technique in both the linac and the bend/folding segments of the machine. Results are benchmarked with the multiparticle tracking code IMPACT to both verify the accuracy of the model and clarify improvements in computational efficiency.
\end{abstract}

DOI: 10.1103/PhysRevSTAB.17.034001

PACS numbers: 29.27.Eg

\section{INTRODUCTION}

The traditional linear envelope model is widely used to model the bundle of linear particle orbits in a linac [1,2]. Instead of tracking every particle and calculating beam parameters like the rms radius, the linear envelope model evaluates the envelope parameters of the beam from the initial quadratic envelope matrix and the transfer matrix between the initial point and the objective point according to Eq. (1) [1]:

$$
\sigma_{2}=R \sigma_{1} R^{T}
$$

Here, $\sigma$ is the $6 \mathrm{D}$ envelope matrix, subscripts 1 and 2 indicate the initial and final states, and $R$ is the transfer matrix between points 1 and 2 .

There are a number of linac codes which are based on or contain this scheme, like TRACE 3D [2]. The advantage of the linear envelope model is obvious: it is fast in computational speed, simple, and provides a clear interpretation of the physics. Therefore, the linear envelope model remains the most preferred model in computationally intensive applications such as global optimization of linac lattices

\footnotetext{
*hez@frib.msu.edu

Published by the American Physical Society under the terms of the Creative Commons Attribution 3.0 License. Further distribution of this work must maintain attribution to the author(s) and the published article's title, journal citation, and DOI.
}

and time restricted applications like on-line beam tuning. The linear model cannot address nonlinear optic effects or their impact on beam quality.

For traditional accelerators, like electron or proton machines, only one charge state is present and the traditional linear envelope model can be directly adopted. However, interest has recently increased in linac accelerators which have the capability of accelerating multiple charge states simultaneously. The Facility for Rare Isotope Beams (FRIB) at Michigan State University [3], for example, is being developed to simultaneously accelerate multiple charge states in order to enhance the total beam power on target. For this new kind of accelerator, the traditional linear envelope model can no longer be adopted directly, and it must be extended to model multicharge state acceleration.

\section{POSSIBLE MULTICHARGE STATE ACCELERATION MODELS}

To analyze the multicharge state acceleration problem, four different schemes are obvious.

First, the most simple and straightforward one is to employ multiparticle tracking codes. In this case, one just treats the particle charge state as a variable instead of a constant and adjusts each lattice element transfer map according to the charge state. The advantages of this scheme are that it requires only minimal changes in existing particle tracking codes, provides detailed information on 
the particle distribution, and it can be applied to analyze nonlinear effects. A standard particle tracking code like IMPACT [4] can be used in this scheme. However, the shortcoming of this method is also obvious: high computational intensity makes the modeling speed too slow for many applications. Moreover, physics results obtained can also be unclearly expressed due to the high detail of the model.

The second approach is to treat charge state deviation as momentum deviation in the magnetic field. The magnetic rigidity is defined as the particle momentum over the charge state. If a charge state is different from a reference charge state, then we find an equivalent momentum by scaling, and adjust the phase-space momentum deviation with respect to the new momentum. This method is exact for the transverse coordinates and can reuse most parts of existing design codes. However, it also has an obvious problem: the method can only handle an all-magnetic field lattice and cannot be applied to the electric field components in rf cavities. The unphysical momentum deviation introduced also causes an unphysical change in the particle time of flight. This can be a severe problem for heavy-ion accelerators where the axial particle velocity is typically far less than speed of light. The scaling method also cannot be written in matrix form, inhibiting use of standard linear envelope model codes. In spite of these limitations, the scaling method can be particularly useful when evaluating the range of multicharge state changes in a bending magnet.

A third approach is to treat the different charge states as a linear deviation from the reference charge state and to extend the usual 6D matrix algebra to 7D matrix algebra. By rewriting the transfer matrix for each element in 7D, we can keep the linear adjustment from the charge state deviation. This method requires significant work to extend common concepts like beam emittance and Courant-Snyder parameters consistently with the influence from 7 th dimension. There are other obvious drawbacks. This method requires new codes or significant modifications to existing codes, so that we cannot make straightforward use of existing resources. Additionally, a linear adjustment in the charge state deviation will not likely always be sufficient. This is especially for charge states which are far away from the reference charge state, where higher-order terms neglected in a linear model can be significant.

\section{A. The three-step scheme}

A fourth approach, which we find to be the preferred one for many purposes, is a three-step scheme where the different charge states are treated separately. This method can be divided into three steps: (i) Use an ideal particle with reference charge state to initiate the machine parameters, such as rf phases, and bending magnet strengths. A record of this reference particle is saved for the whole beam. (ii) Choose particles located at the beam center of each charge state as secondary reference particles and carry out single particle tracking simulations according to the initialized lattice parameters. Keep records of each secondary particle and make it the reference for each charge state of the beam. (iii) Apply the linear envelope model for each charge state bunch of the beam. The transfer matrix should be adjusted consistently with the associated charge state and reference orbit.

This method of handling multicharge state has a balance between the high fidelity of the particle tracking method and the efficiency of the linear envelope model. Separating each charge state helps make the physics simple and clear. Higher-order effects caused by charge state differences during bending can be smaller when the beam size for each charge state is relatively smaller compared with dispersive effects. Codes developed for both particle tracking and linear envelope models can be reused without modification. Because particle tracking simulations are carried out only for a limited set of reference particles, computational efficiency is also high provided that not too many charge states are accelerating simultaneously-which is true for most applications.

Application details of the three-step scheme can be different according to the specific machine and codes employed. However, the three-step scheme is universal and can be adapted to every multicharge accelerator.

\section{B. rms envelope calculation}

In most conceivable experimental applications, diagnostic limitations will not allow the rms size of the beam to be measured separately for each charge state. In order to apply the three-step scheme to measure the rms beam size including all species, results for each charge state must be recombined for consistent calculation of the total rms beam size. In the center-of-mass frame, $\bar{x}$ represents the center of the beam for each charge state, $\sigma$ represents the rms size, $N$ is the particle number, subscript $i$ denotes different charge states ( $N$ total particles, $N_{i}$ particles in species $i$ ). Then the rms beam size of the full beam is given by

$$
\sigma=\sqrt{\frac{\sum_{i} N_{i}\left(\sigma_{i}^{2}+\bar{x}_{i}^{2}\right)}{N}} .
$$

After the recombination, the rms beam size of the full beam can be compared with results from particle tracking codes and/or experiment to benchmark the three-step scheme. Henceforth, the "multicharge state envelope model" will refer to the three-step scheme outlined.

\section{APPLICATION TO A FODO LATTICE}

Before analyzing a complex lattice, a quadrupole FODO (focus-drift-defocus-drift) focusing lattice is used as an example to test the multicharge state envelope model.

The test lattice is a simple FODO quadrupole channel consisting of only drift spaces and quadrupoles. The lattice 
period is 8 meters long consisting of 3 meters of drift space followed by 1 meter long quadrupole (focusing strength $0.5 \mathrm{~T} / \mathrm{m}$ with no fringe effects), then followed by another 3 meters of drift space and another 1 meter long defocusing quadrupole (focusing strength $-0.5 \mathrm{~T} / \mathrm{m}$ ). Four lattice periods are simulated with a final 3-meter drift space (corresponding to a total lattice length of 35 meters). The usual drift space and thick quadrupole transfer matrices are used. A uranium beam with five charge states, $76+$, $77+, 78+, 79+$, and $80+$, and an axial kinetic energy $16.475 \mathrm{MeV} / \mathrm{u}$ is injected into the lattice. The initial $\mathrm{X}$-direction phase-space distribution injected is shown in Fig. 1. Each charge state beam is Gaussian distributed in position and angle with an rms width of around $1.5 \mathrm{~mm}$ and an rms angle of around $0.5 \mathrm{mrad}$. All species are initially coincident axially. The beam centers of neighboring charge states are separated by a distance of around $3 \mathrm{~mm}$. Both the multicharge state envelope model and the IMPACT code are used to model the beam.

The multicharge state envelope model provides information on both the beam center evolution and the rms beam size evolution of the five charge states. Results are presented in Fig. 2.

Results from the rms envelope recombination scheme [see Eq. (2)] and the IMPACT particle tracking code for the rms width of the full beam are compared in Fig. 3. Curves obtained from the multicharge state envelope model and IMPACT agree well. Results should, in principle, be identical since the FODO lattice as modeled is purely linear. However, small differences result from numerical errors due to differences associated with the finite step size employed in the envelope code and IMPACT as well as noise associated with finite particle statistics used to represent the distribution in IMPACT. In this sense, the test is not very demanding, but serves the purpose of showing there are no setup problems in the envelope model.

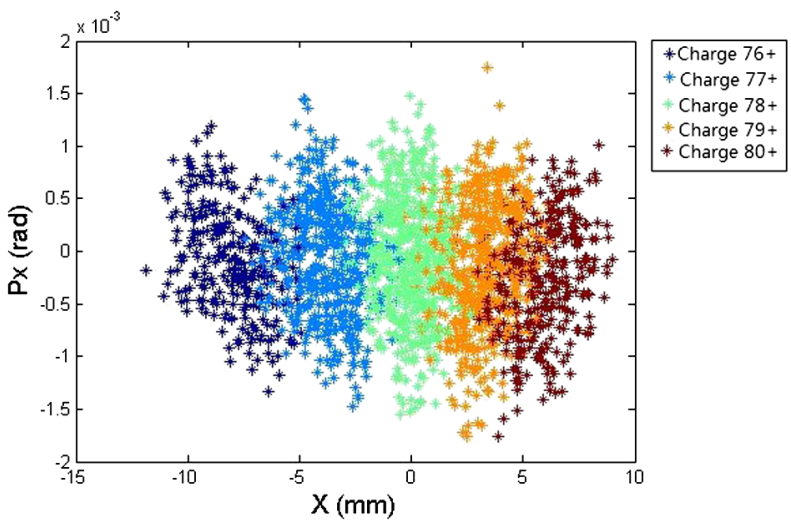

FIG. 1. Initial X-direction phase-space distribution used to test the multicharge state envelope model in a FODO transport lattice. The beam has five charge states (color coded), whose beam centers are transversely separated relative to each other.

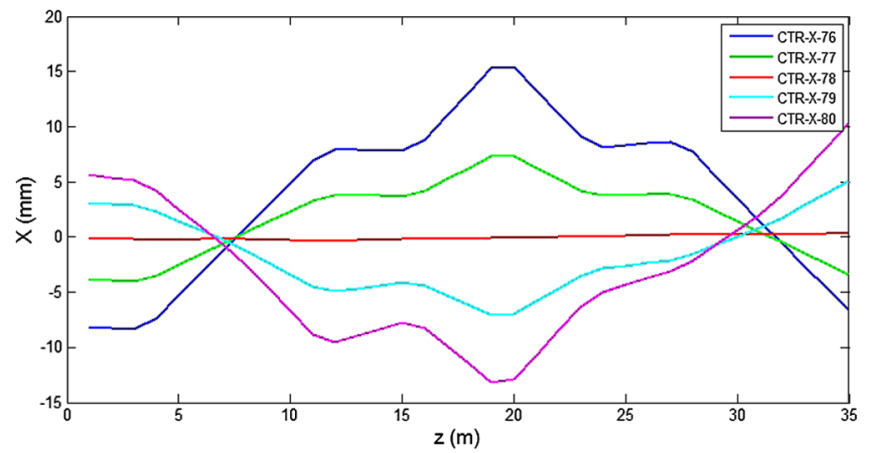

(a)

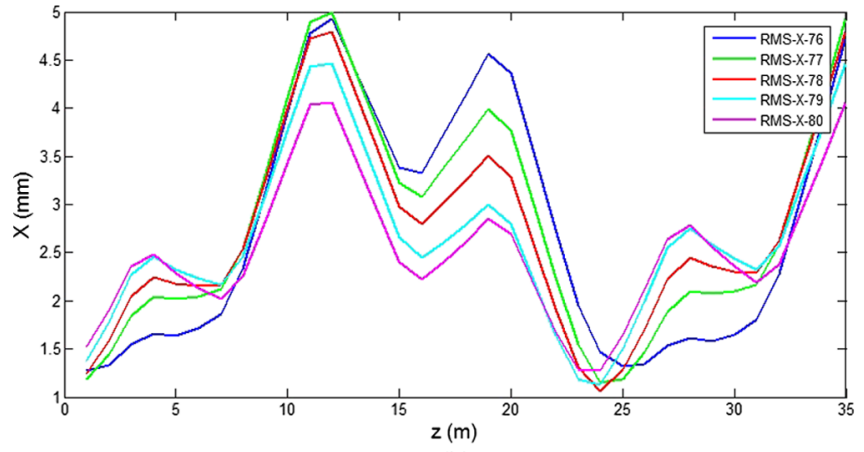

(b)

FIG. 2. Multicharge state envelope model results for the test FODO lattice and the initial uranium beam with five charge states given in Fig. 1. (a) X-direction beam center evolution, and (b) X-direction beam rms envelope evolution.

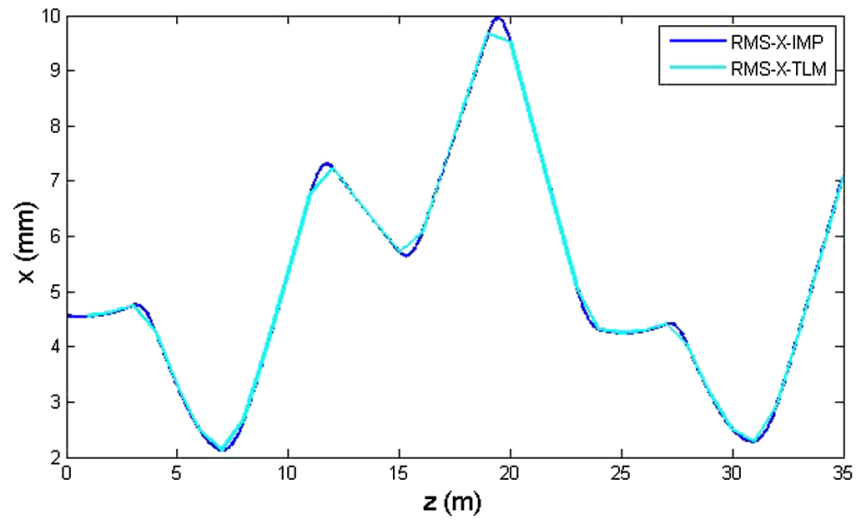

FIG. 3. Contrast of the X-direction rms envelope widths calculated for the full beam by the multicharge state envelope model and the IMPACT particle tracking code for the example FODO lattice with the initial particle distribution in Fig. 1.

\section{APPLICATION TO FRIB}

The FRIB accelerator presently under construction at Michigan State University will deliver stable ion beams with energy more than $200 \mathrm{MeV} / \mathrm{u}$ with continuous, ontarget beam power of more than $400 \mathrm{~kW}$ [3,5]. In order to meet this goal, multicharge state acceleration is needed, 


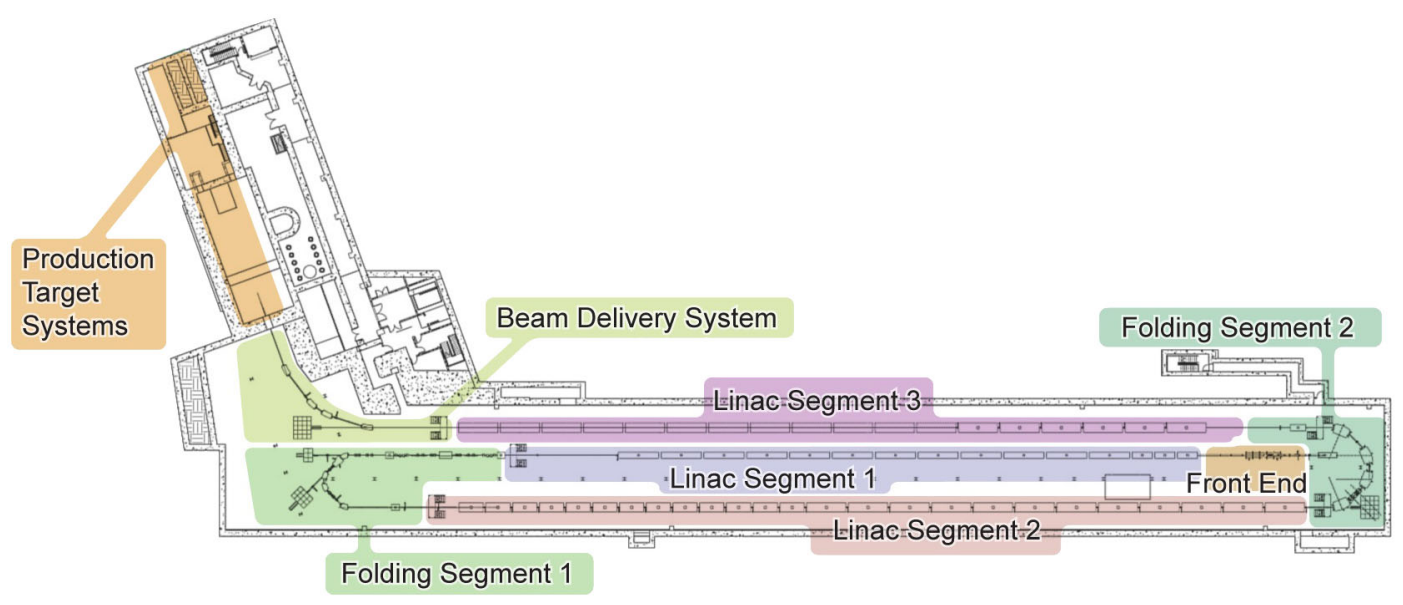

FIG. 4. FRIB lattice.

especially for heavy ions, to increase the beam current and beam power. Figure 4 shows the layout of FRIB lattice. The FRIB accelerator consists of a front-end segment, three linac segments, two folding segments and an on-target beam delivery system. Uranium, 33+, 34+ charge states are accelerated in linac segment 1 , and after a charge stripper and a charge selector at the folding segment 1 , $76+, 77+, 78+, 79+, 80+$ charge states emerging from the stripper are selected and accelerated all the way to the target. Careful machine optimization is required to minimize potential particle losses in the high-power beam. This will require much effective modeling of the many potential operating modes of the machine necessitating development of simple, but high-fidelity models.

\section{A. Linac segments}

The linac segments are where heavy ions gain most of their kinetic energy. Detailed information on the FRIB lattice can be found in Ref. [5]. For linac segment 1, two kinds of superconducting quarter-wave resonators (QWRs), with optimum beta values of 0.041 and 0.085 , are used to accelerate the ion species. For linac segment 2, two kinds of superconducting half-wave resonators with optimum beta values of 0.29 and 0.53 are used. For both linac segment 1 and linac segment 2, superconducting solenoids provide the main transverse focusing strength and limited focusing is also provided by the rf cavities.

Linear matrix models. - To track the energy and phase advance for the synchronous particle in an rf cavity, the drift-kick-drift thin-lens model given by [1]

$$
\begin{aligned}
W_{2} & =W_{1}+q V T(k) \cos \varphi_{1}-q V S(k) \sin \varphi_{1}, \\
\varphi_{2} & =\varphi_{2}+\frac{q V}{2 W_{1}} k\left[T^{\prime}(k) \sin \varphi_{1}+S^{\prime}(k) \cos \varphi_{1}\right]
\end{aligned}
$$

is employed. Here, $W$ denotes the particle kinetic energy, $\varphi$ the longitudinal phase, $q$ is the particle charge, and $V$ is amplitude of the rf voltage. Subscripts 1 and 2 indicate the initial and final states. $T, T^{\prime}, S, S^{\prime}$ are transit time factors which are defined by

$$
\begin{aligned}
T(k) & =\frac{\int_{a}^{b} E_{z}(z) \cos k z d z}{\int_{a}^{b} E_{z}(z) d z}, \\
S(k) & =\frac{\int_{a}^{b} E_{z}(z) \sin k z d z}{\int_{a}^{b} E_{z}(z) d z}, \\
T^{\prime}(k) & =\frac{d T(k)}{d k}=\frac{\int_{a}^{b} z E_{z}(z) \sin k z d z}{\int_{a}^{b} E_{z}(z) d z}, \\
S^{\prime}(k) & =\frac{d S(k)}{d k}=\frac{\int_{a}^{b} z E_{z}(z) \cos k z d z}{\int_{a}^{b} E_{z}(z) d z} .
\end{aligned}
$$

The traditional drift-kick-drift thin-lens model assumes constant velocity when calculating energy gain. However, the assumptions associated with the model are not exact, especially for low energy beams. To decrease this error, the two accelerating gaps are treated separately for the QWRs instead of treating the whole cavity as a single accelerating gap [6].

The transfer matrix for the longitudinal phase space is obtained from the drift-kick-drift model. By differentiating the longitudinal drift-kick-drift thin-lens model and keeping the linear term in the energy and phase deviation, we obtain

$$
\left[\begin{array}{l}
\Delta \varphi_{2} \\
\Delta E_{2}
\end{array}\right]=\left[\begin{array}{cc}
1 & 0 \\
-q V_{0} T \sin \varphi_{1}-q V_{0} S \cos \varphi_{1} & 1
\end{array}\right]\left[\begin{array}{l}
\Delta \varphi_{1} \\
\Delta E_{1}
\end{array}\right] .
$$

The transfer matrix of the transverse phase space also comes from a drift-kick-drift thin-lens model. Phase-space coordinates $x, x^{\prime}, y, y^{\prime}$ are used. For each acceleration gap, the total transfer matrix is decomposed into drift spaces between two focusing/defocusing gaps and an acceleration gap which gives 


$$
M_{\mathrm{trans}}=M_{\mathrm{drf}} M_{F} M_{\mathrm{drf}} M_{\mathrm{acc}} M_{\mathrm{drf}} M_{F} M_{\mathrm{drf}} .
$$

Here, $M_{\text {drf }}$ is the matrix for an $\mathrm{rf}$ drift space, $M_{F}$ is the transfer matrix for $\mathrm{rf}$ focusing defined by

$$
M_{F}=\left[\begin{array}{cccc}
1 & 0 & 0 & 0 \\
k_{F} & 1 & 0 & 0 \\
0 & 0 & 1 & 0 \\
0 & 0 & k_{F} & 1
\end{array}\right]
$$

with

$$
k_{F}=\frac{q V_{F}\left(T_{F} \cos \varphi-S_{F} \sin \varphi\right)}{\beta^{2} \gamma E_{s}}
$$

denoting the transverse focusing/defocusing gap strength, $V_{F}$ is the integrated focusing/defocusing voltage, $T_{F}$ and $S_{F}$ are the transverse focusing/defocusing gap transit time factors, $\varphi$ is rf phase at the gap, and $E_{s}=m_{0} c^{2}$ is rest energy of the particle. The transfer matrix $M_{\text {acc }}$ describes the focusing effect due to longitudinal acceleration:

$$
\begin{aligned}
M_{\mathrm{acc}} & =\left[\begin{array}{llll}
1 & 0 & 0 & 0 \\
0 & a & 0 & 0 \\
0 & 0 & 1 & 0 \\
0 & 0 & 0 & a
\end{array}\right], \\
a & =\frac{(\beta \gamma)_{1}}{(\beta \gamma)_{2}}
\end{aligned}
$$

where $(\beta \gamma)_{1}$ is the initial $\beta \gamma$ before acceleration and the $(\beta \gamma)_{2}$ is the final $\beta \gamma$ after acceleration.

A soft-edge solenoid model [7] is applied with

$$
M_{\text {softsol }}=M_{\text {edge }} M_{\text {sol }} M_{\text {edge }} .
$$

Here,

$$
M_{\mathrm{sol}}=\left[\begin{array}{cccc}
\cos ^{2} \theta & \frac{\sin \theta}{g} & \sin \theta & \frac{\sin ^{2} \theta}{g} \\
-g \sin \theta & \cos ^{2} \theta & -g \sin ^{2} \theta & \sin \theta \\
-\sin \theta & -\frac{\sin ^{2} \theta}{g} & \cos ^{2} \theta & \frac{\sin \theta}{g} \\
g \sin ^{2} \theta & -\sin \vartheta & -g \sin \theta & \cos ^{2} \theta
\end{array}\right]
$$

is the traditional hard edge solenoid transfer matrix where $g=\frac{B q c}{2 \beta E_{\mathrm{tot}}}, \theta=g L$, and

$$
M_{\text {edge }}=\left[\begin{array}{cccc}
1 & 0 & 0 & 0 \\
-\Phi & 1 & 0 & 0 \\
0 & 0 & 1 & 0 \\
0 & 0 & -\Phi & 1
\end{array}\right]
$$

describes the edge effect where $\Phi=-\frac{g^{2} a}{2}$ ( $g$ is the solenoid focusing strength and $a$ is the solenoid radius).

These element models have been verified using a single charge state with the IMPACT tracking code, and can be extended to handle multicharge state acceleration by adopting the three-step scheme.

Linac segment 1.-The first step is to initialize the lattice parameters using a reference charge state. For the linac segment 1 case where the two charge states are $33+$ and $34+$, either one can be employed as reference. Here we choose $33+$ as the reference and the lattice is initialized using an ideal particle. The rf cavity phases are set so that the ideal particle has the designed synchrotron phase and gets accelerated to the designed energy. The solenoid field strengths are set so that the ideal particle has the designed focusing strength.

After initializing the lattice parameters, the center particles for $33+$ and $34+$ charge state beam bunches are tracked and results are recorded as reference orbits. The initial distribution employed was imported from a detailed simulation of the FRIB front end by TRACK [8]. The distribution is imported into the IMPACT code and moments calculated from it are used to initialize the envelope code. The initial X-direction and Z-direction phase-space projections of the distribution are shown in Fig. 5. The Y-direction phase-space projection is analogous with the $\mathrm{X}$-direction.

After this step, the envelope model is used to track the envelopes of the $33+$ and $34+$ charge state beam bunches separately. Transfer matrices are influenced not only by charge state but also by the reference orbit. For example, the transverse and longitudinal transfer matrix of rf cavity is a function of synchrotron phase, and this phase is different for the different charge states.

After finishing these three steps, we obtain both beam centers and beam envelopes for the $33+$ and $34+$ charge states. The longitudinal phase deviation and rms envelopes of the two charge states in the center-of-mass frame are given in Fig. 6(a). Note that the 33+ charge state particle tends to have a larger phase than the $34+$ charge state. This occurs because the $33+$ charge state cannot be accelerated as efficiently as the $34+$ charge state, and would always lag behind a little to gain the same energy. The oscillation in longitudinal phase amplitude for both charge states is larger than their longitudinal rms envelope size, which means that
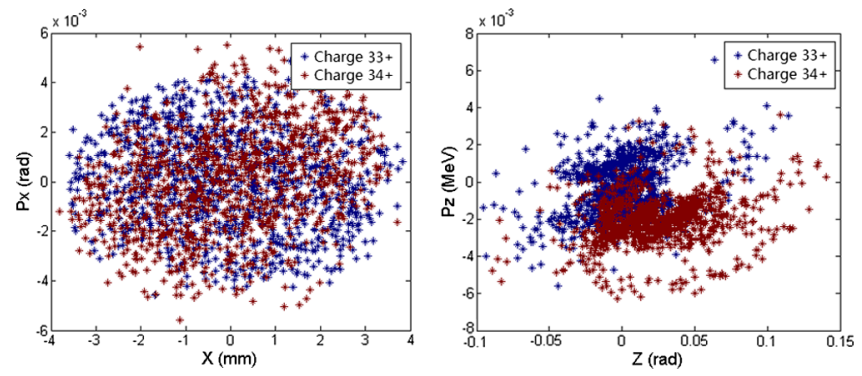

FIG. 5. Initial X-direction and Z-direction phase-space projections of the distribution at the entrance of FRIB linac segment 1. 


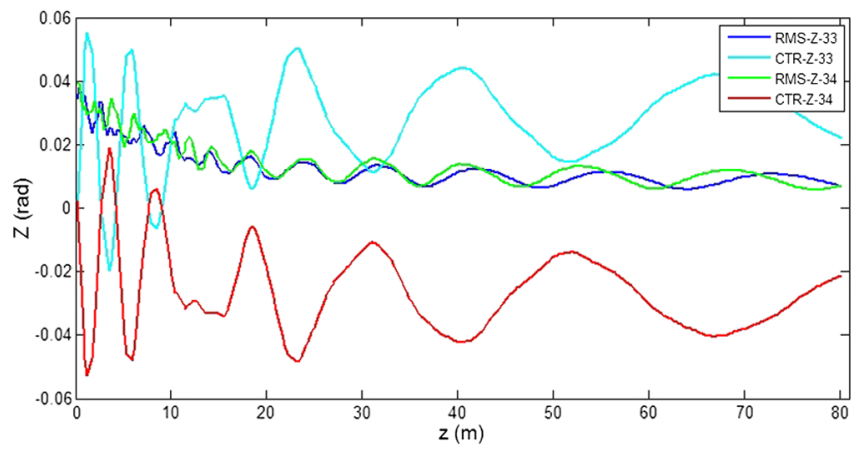

(a)

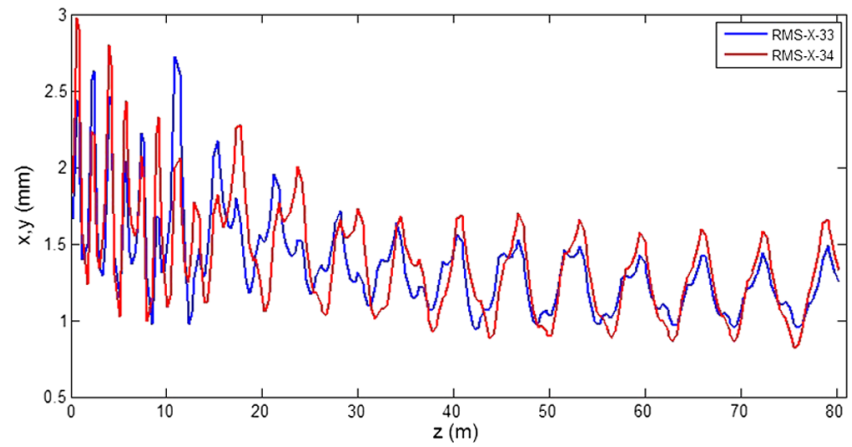

(b)

FIG. 6. FRIB linac segment 1 envelope model results. (a) Longitudinal phase center deviation for the $33+$ and $34+$ charge states and the rms envelope of the phase spread in the center-of-mass frame. (b) Transverse beam rms envelope for the $33+$ and $34+$ charge states.

adding another charge state would dramatically increase the longitudinal extent of the full beam. Figure 6(b) shows results for the transverse size of each charge state of the beam. The center of the beam for each charge state is coincident with the machine axis for the distribution analyzed. Due to the different focusing strengths for the different charge states, the focusing lattice cannot match both charge states at the same time, so we can only minimize the total beam size. Consequently, rms envelope mismatch oscillations occur for both charge states.

By using the rms envelope recombination scheme in Eq. (2), the rms beam size obtained in the multicharge state envelope model is benchmarked against results obtained from the multiparticle tracking code IMPACT. Results in Fig. 7 show good agreement between the models. Small differences between the models in this case result not only from numerical differences as described in the FODO example in Sec. III but also nonlinear effects coming from longitudinal acceleration included in IMPACT.

Linac segment 2.-A similar three-step scheme process is applied to the FRIB linac segment 2. For this case, 76+, $77+, 78+, 79+, 80+$ charge states are accelerated for the uranium beam and the center charge state $78+$ is chosen as the reference. Results for beam centers and envelopes

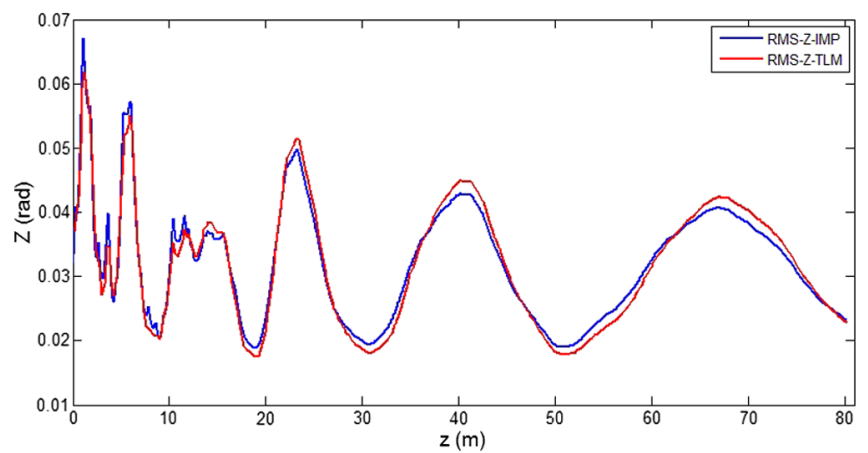

(a)

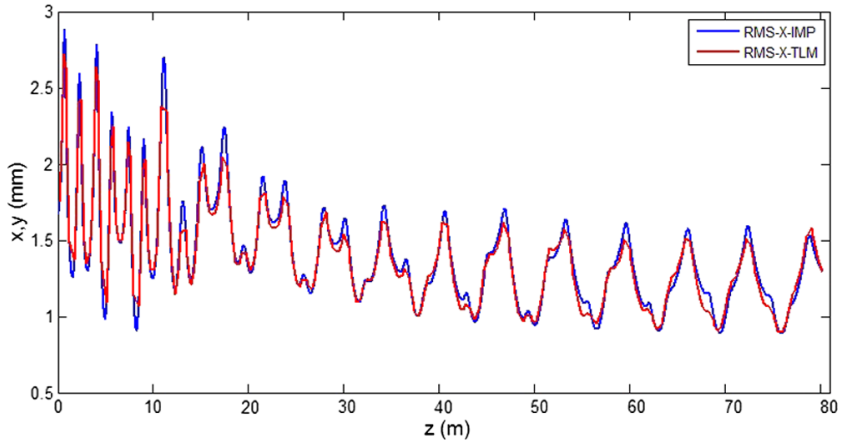

(b)

FIG. 7. FRIB linac segment 1 results for the full-beam rms envelope calculated from the envelope model and IMPACT. Envelopes for the longitudinal plane (a) and the transverse plane (b).

of the five charge states are given in Fig. 8. Similar to linac segment 1 case, we can see from Fig. 8(a) that beam centers of longitudinal phase of the five different charge states eventually separate and damp to their own corresponding steady points due to their different longitudinal acceleration efficiencies. From Figs. 8(b) and 8(c), we see that different charge states result in different longitudinal and transverse focusing strength, thereby causing the rms envelope evolution of each charge state to be a little different relative to each other.

Similar to linac segment 1 case, we apply the rms envelope recombination scheme in Eq. (2) and compare results with the IMPACT code. Results given in Fig. 9 again indicate good agreement between the envelope and full particle tracking models. Small deviations occur for analogous reasons to those discussed for linac segment 1 .

\section{B. Folding segment}

There are two 180 degree bending segments linking the three linac segments of FRIB. Except for components like quadrupoles, which can be described by the common quadrupole transfer matrix, and rebunching rf cavities (a set of rf cavities with -90 degree synchrotron phase), which have been discussed in the analysis of linac 


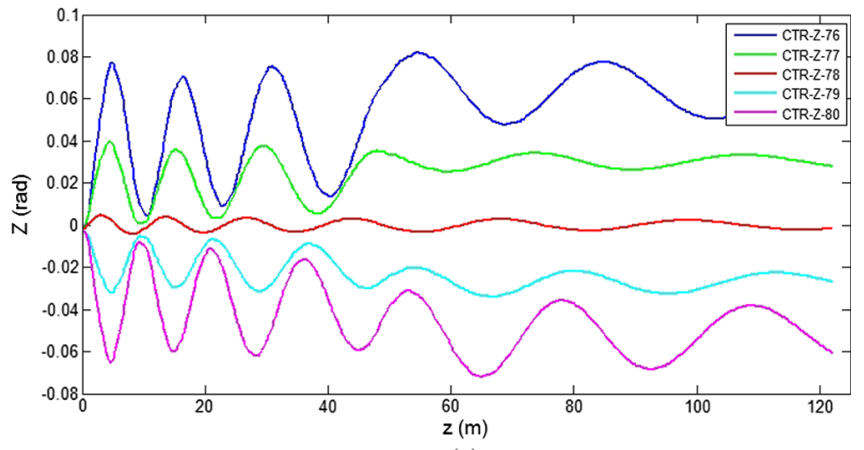

(a)

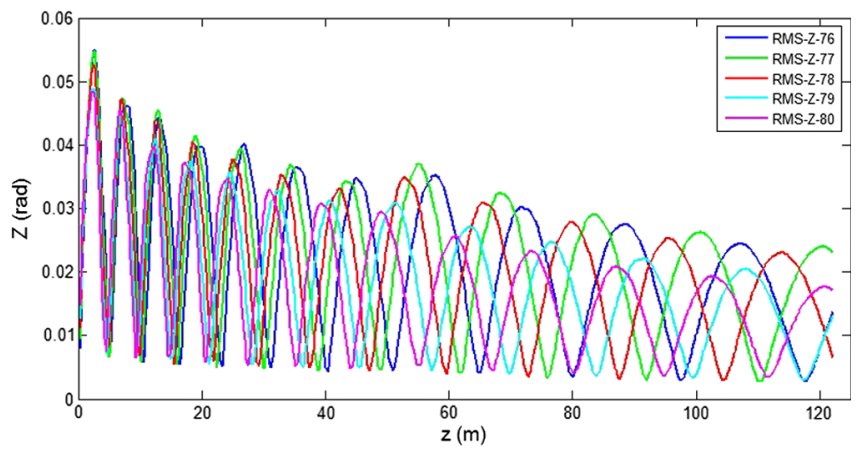

(b)

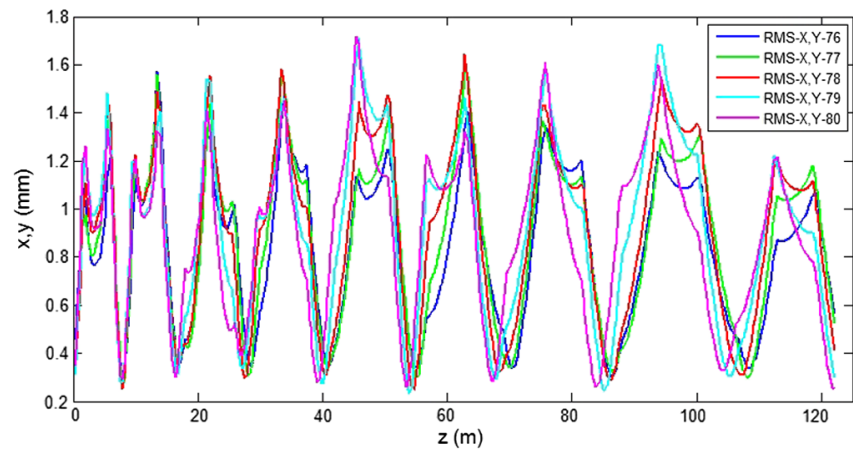

(c)

FIG. 8. Envelope model results for FRIB linac segments 2 for the five charge states transported. (a) Evolution of the longitudinal center phase. (b) Longitudinal rms phase width. (c) Transverse rms envelope extent.

segment 1 , the most crucial components are the bending magnets. For a charge state far away from reference whose dispersion is high, higher-order terms may appear and compromise the accuracy of the linear transfer matrix. In

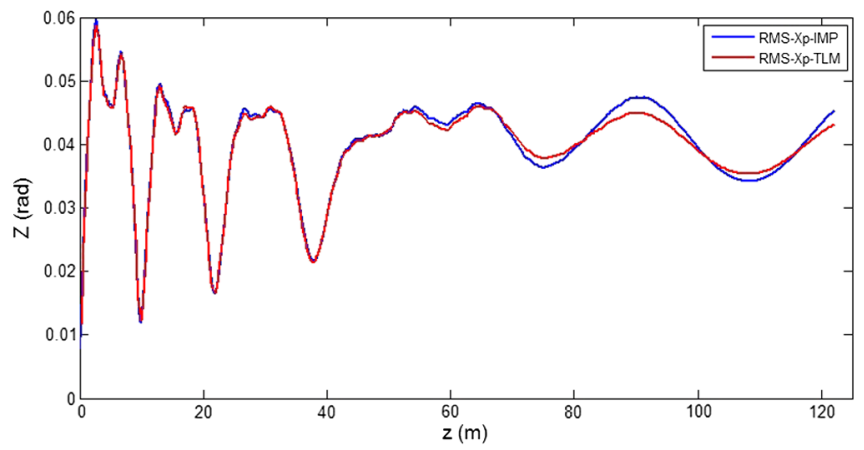

(a)

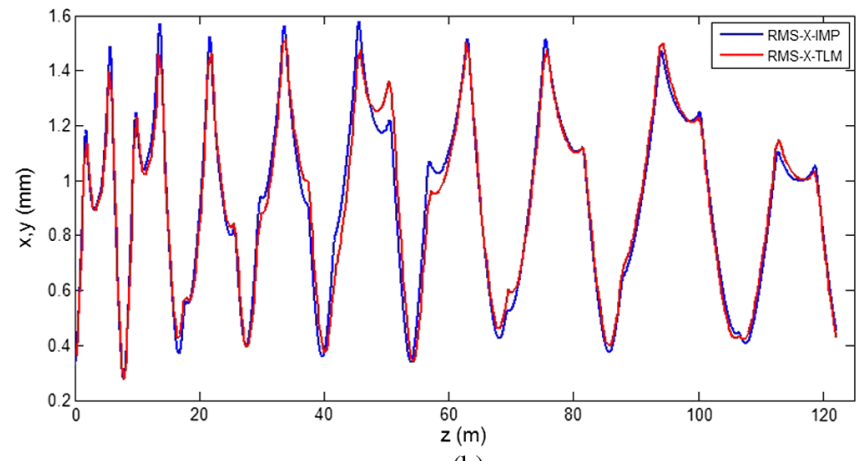

(b)

FIG. 9. FRIB linac segment 2 results for the full beam based on the envelope model and IMPACT simulations. (a) Full-beam rms envelope for the longitudinal plane. (b) rms envelope for the transverse plane.

order to include the potential influence from higher-order terms, a new scheme has been proposed to handle multicharge state transport in a bending magnet.

First, we employ a reference charge state to initialize the lattice parameters. For the uranium beam in folding segment 1 , where the charge states are $76+, 77+, 78+, 79+$, and $80+$, the charge state $78+$ is chosen to initialize the lattice parameters; mainly the phase for rebunching cavities, quadrupole magnet field strengths, and bending magnet field strengths. Then, for the center particles of the bunches of the five charge states, their reference orbits are calculated. In order to include possible higher-order terms coming from bending magnets, the exact transfer map for an ideal sector bending magnet is used to track the reference particles [9]:

$$
\begin{aligned}
& x_{2}(s)=\frac{\rho}{b}\left(\frac{1}{\rho} \sqrt{\left(1+\delta_{1}\right)^{2}-x_{2}^{\prime}(s)^{2}-y_{1}^{\prime 2}}-\frac{d x_{2}^{\prime}(s)}{d s}-b\right), \quad x_{2}^{\prime}(s)=x_{1}^{\prime} \cos \left(\frac{s}{\rho}\right)+\left[\sqrt{\left(1+\delta_{1}\right)^{2}-x_{1}^{\prime 2}-y_{1}^{\prime 2}}-b\left(\rho+x_{1}\right)\right] \sin \left(\frac{s}{\rho}\right), \\
& y_{2}(s)=y_{1}+\frac{y_{1}^{\prime} s}{b \rho}+\frac{y_{1}^{\prime}}{b}\left[\sin ^{-1}\left(\frac{x_{1}^{\prime}}{\sqrt{\left(1+\delta_{1}\right)^{2}-y_{1}^{\prime 2}}}\right)-\sin ^{-1}\left(\frac{x_{2}^{\prime}(s)}{\sqrt{\left(1+\delta_{1}\right)^{2}-y_{1}^{\prime 2}}}\right)\right], \quad y_{2}^{\prime}(s)=y_{1}^{\prime}, \\
& l_{2}(s)=l_{1}+\frac{\left(1+\delta_{1}\right) s}{b \rho}+\frac{1+\delta_{1}}{b}\left[\sin ^{-1}\left(\frac{x_{1}^{\prime}}{\sqrt{\left(1+\delta_{1}\right)^{2}-y_{1}^{\prime 2}}}\right)-\sin ^{-1}\left(\frac{x_{2}^{\prime}(s)}{\sqrt{\left(1+\delta_{1}\right)^{2}-y_{1}^{\prime 2}}}\right)\right], \quad \delta_{2}(s)=\delta_{1} .
\end{aligned}
$$




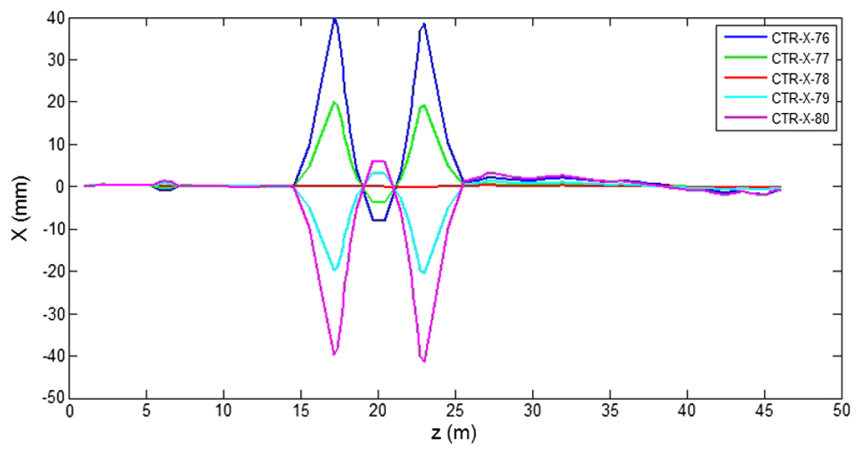

(a)

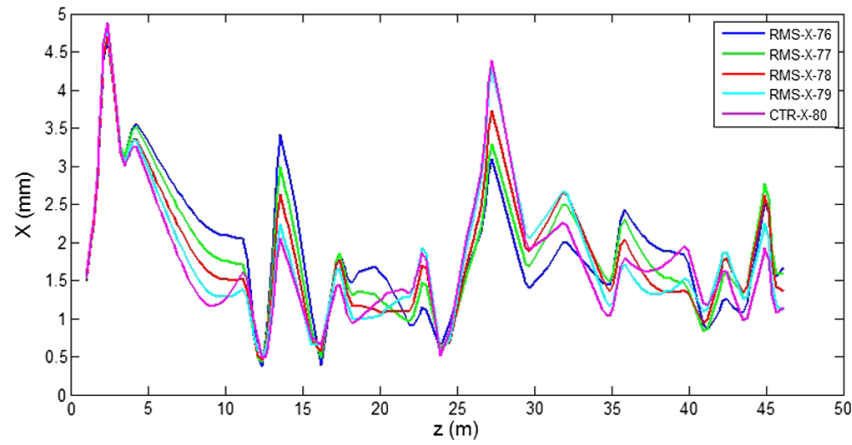

(b)

FIG. 10. Envelope simulation results for the five charge states in the FRIB folding segment 1. (a) Transverse beam centers. (b) rms envelope.

Here, $\rho$ is the radius of curvature of the bend, $b$ is the radius of magnetic curvature, $\left(x_{1}, x_{1}^{\prime}, y_{1}, y_{1}^{\prime}, l_{1}, \delta_{1}\right)$ are the initial 6D phase-space coordinates, and $\left[x_{2}(s), x_{2}^{\prime}(s)\right.$, $\left.y_{2}(s), y_{2}^{\prime}(s), l_{2}(s), \delta_{2}(s)\right]$ are the final phase-space coordinates at downstream distance $s$ from the original point. $\delta$ and $l$ can be derived from $\Delta \varphi$ and $\Delta E . x_{2}^{\prime}(s)$ is calculated first before calculating other phase space coordinates which depend on $x^{\prime}(s)$ [e.g., $x(s), y(s)$, and $l(s)$ ]. After calculating the reference orbits, we apply the envelope model to analyze the envelope of the species. The traditional bending magnet transfer matrix for an ion beam can be used [10]:

$$
M_{\text {bend }}=\left[\begin{array}{cccccc}
C & \frac{s}{h} & 0 & 0 & 0 & \frac{1-C}{h} \\
-h S & C & 0 & 0 & 0 & S \\
0 & 0 & 1 & L & 0 & 0 \\
0 & 0 & 0 & 1 & 0 & 0 \\
S & \frac{1-C}{h} & 0 & 0 & 1 & L-\frac{s}{h} \\
0 & 0 & 0 & 0 & 0 & 1
\end{array}\right] .
$$

Here, the phase-space coordinate is also $\left(x, x^{\prime}, y, y^{\prime}, l, \delta\right)$; $C=\cos \alpha, S=\sin \alpha, h=1 / \rho$, where $\alpha$ is the bending angle, and $\rho$ is the geometry curvature. A two-wedge transfer matrix should also be added according to the entrance and exit pole face angles [10]. Note that the entrance and exit pole-face angles $\alpha$ and $\rho$ should be adjusted according to the reference orbits of the different

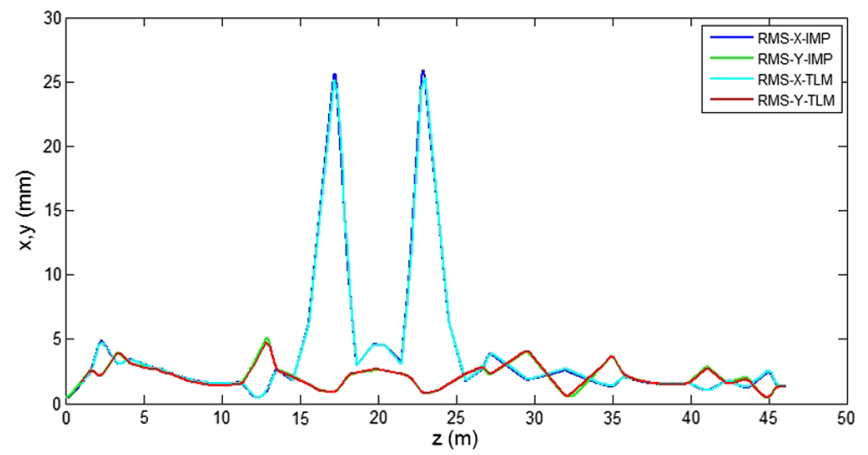

FIG. 11. FRIB linac folding segment results for the full transverse beam envelope in the FRIB folding segment 1. Envelope model and IMPACT results are contrasted.

charge states. Care must be taken since the ion beam is usually nonrelativistic, and timing of the species should be taken into consideration when calculating longitudinal phase from the path length.

Using the three-step scheme, we present the beam center and rms size of each charge state in folding segment 1 in Fig. 10. From Fig. 10(a) we note that after a bend, the transverse centers of the five charge states separate because of dispersive effects. The FRIB bending lattice is designed so that beam centers of the different charge states recombine with each other after the folding segment. Figure 10(b) indicates that the rms beam size varies according to the charge state. At high dispersion points, the beam size is dominated by dispersion. At this point, distance between nearby charge states can be as high as $20 \mathrm{~mm}-10$ times larger than the corresponding rms envelope size. So different charge states can be completely separated at such points, which can provide good locations to place charge selectors or beam collimators.

Full beam envelope results obtained by applying the recombination scheme in Eq. (2) and comparing results with IMPACT are shown in Fig. 11. Aspects of the thin-lens model employed also agree well with the IMPACT result in benchmark tests carried out for the folding segment.

\section{CONCLUSION}

The multicharge state envelope model developed has been shown to be both precise and numerically efficient. Results obtained agree well with those from multiparticle tracking codes. Only minor extensions of currently available codes for linear envelope models are needed. The computational efficiency is high. A typical beginning-toend computation of the FRIB lattice employing the multiparticle tracking code IMPACT takes several minutes on available computer systems at acceptable resolutions, whereas the multicharge state envelope model takes only several seconds. This large enhancement in computational efficiency makes the model promising for on-line applications like fast beam tuning and lattice optimization for complex multicharge state machines such as FRIB. 


\section{ACKNOWLEDGMENTS}

The authors would like to thank J. Qiang, the author of the IMPACT code, for useful guidance on using IMPACT and understanding the physics models contained in the code. Also, we wish to acknowledge useful discussions with C. Tang from the Accelerator Lab at Tsinghua University; Y. Yamazaki, F. Marti, and S. Lund from FRIB at Michigan State University. This work was supported by the U.S. National Science Foundation under Grant No. PHY-11-02511, and the U.S. Department of Energy Office of Science under Cooperative Agreement No. DE-SC0000661.

[1] T. P. Wangler, Principles of RF Linear Accelerators (John Wiley \& Sons, New York, 1998).
[2] K. R. Crandall and D. P. Rusthoi, Trace 3-D, Los Alamos National Lab [http://laacg.lanl.gov/laacg/services/traceman .pdf].

[3] J. Wei et al., in Proceedings of HIAT 2012, Chicago, IL USA, pp. 8-19.

[4] J. Qiang et al., J. Comput. Phys. 163, 434 (2000).

[5] R. C. York et al., in Proceedings of the 46th ICFA Advanced Beam Dynamics Workshop on High-Intensity and High-Brightness Hadron Beams HB2010, Morschach, Switzerland, 2010 (PSI, Viligen, Switzerland, 2011), pp. 319-323.

[6] Z. He et al., in Proceedings of PAC2013, Pasadena, CA USA, pp. 609-611.

[7] M. Aslaninejad, in Proceedings of IPAC'10, Kyoto, Japan, pp. 3963-3965.

[8] V. N. Aseev, P. N. Ostroumov et al., TRACK code, Argonne National Lab [http://www.phy.anl.gov/atlas/TRACK/].

[9] E. Forest, Beam Dynamics, A New Attitude and Framework (Harwood Academic, Chur, Switzerland, 1998), p. 360.

[10] K. L. Brown, Report No. SLAC-75, 1982. 